\title{
Adrenomedullin augments the neurohumoral response to haemorrhage in non-pregnant but not in pregnant sheep
}

\author{
C J Charles, M T Rademaker, A M Richards and M G Nicholls \\ Christchurch Cardioendocrine Research Group, Christchurch School of Medicine, Christchurch, New Zealand \\ (Requests for offprints should be addressed to C J Charles, Christchurch Cardioendocrine Research Group, Christchurch School of Medicine, PO Box 4345, \\ Christchurch, New Zealand; Email: chris.charles@chmeds.ac.nz)
}

\begin{abstract}
Adrenomedullin (ADM) is a novel peptide with actions which include reduction of arterial pressure and interaction with a number of hormone systems. In order to assess possible interactions with the renin-angiotensin system (RAS) and the hypothalamo-pituitary-adrenal (HPA) axis, we have examined neurohumoral responses to hypotensive haemorrhage $(15 \mathrm{ml} / \mathrm{kg}$ over $15 \mathrm{~min})$ with or without co-infusions of ADM $(5 \cdot 5 \mathrm{pmol} / \mathrm{kg}$ per min) in six non-pregnant and eight pregnant conscious sheep. Haemorrhage induced a greater decrease in arterial pressure, but a blunted increase in heart rate in pregnant sheep. There was no significant effect of ADM on haemodynamic responses to haemorrhage in either group. In
\end{abstract}

non-pregnant sheep, haemorrhage-induced activation of both RAS and HPA was significantly augmented by ADM, as indicated by greater increases in plasma renin activity $(P<0 \cdot 01)$, angiotensin II $(P<0.05)$ and arginine vasopressin $(P<0 \cdot 01)$. In contrast, ADM did not augment these responses to haemorrhage in pregnant sheep. Rather, plasma concentrations of aldosterone $(P=0.039)$ and adrenocorticotrophic hormone $(P=0 \cdot 012)$ were decreased by ADM. In conclusion, ADM-induced augmentation of the RAS and HPA responses to hypotensive haemorrhage is abolished in the pregnant state.

Journal of Endocrinology (2001) 171, 363-371

\section{Introduction}

Adrenomedullin (ADM) is a vasodilator peptide first isolated from human phaeochromocytoma cells and subsequently identified in many tissues, including the adrenal, heart, lung and kidney (Richards et al. 1996). Results from our laboratory show that ADM mRNA is also strongly expressed in the uterus, ovary and posterior pituitary of rats and mice (Cameron \& Fleming 1998). Expression of specific binding sites for ADM have been reported in the uterus, with enhancement of both receptors and the peptide observed in pregnancy (Upton et al. 1997). Further, plasma ADM concentrations are increased in human pregnancy (Di Iorio et al. 1997, 1999, Minegishi et al. 1999). Recent evidence suggests that ADM might have an essential role in fetal development, in that replacing the coding region of the $A D M$ gene in mice resulted in embryos dying at midgestation, with extreme hydrops fetalis and cardiovascular abnormalities (Caron \& Smithies 2001). Taken together, these results suggest that ADM may play a vital part in human reproduction and fetal development.

Biological actions attributed to ADM include clear-cut and sustained reduction of arterial pressure in a number of species, including sheep (Parkes \& May 1995, Charles et al. 1997) and humans (Lainchbury et al. 1997).
Endocrine actions documented in vitro include stimulation of renin release (Jensen et al. 1997), inhibition of angiotensin II-induced and potassium-induced (but not adrenocorticotrophic hormone (ACTH)-induced) aldosterone secretion (Yamaguchi et al. 1994, Nussdorfer et al. 1997, Andreis et al. 1998) and inhibition of ACTH secretion (Samson et al. 1995). Some of these effects have also been reported in vivo, including increased plasma renin activity (PRA) (Charles et al. 1997), inhibition of endogenous (Rademaker et al. 1997) and exogenous angiotensin II(but not ACTH-) stimulated plasma aldosterone (Petrie et al. 2000) and reduction of plasma ACTH and cortisol (Parkes \& May 1995). However, there are no reports of the integrated effects of ADM on both the reninangiotensin system (RAS) and the hypothalamopituitary-adrenal (HPA) systems in response to stress or during pregnancy in conscious animals. Further, the actions of ADM on hormonal axes in pregnancy are unknown. Accordingly, we have assessed possible modulatory actions of ADM on the neurohumoral response to a standardised stress. Thus we examined the haemodynamic and hormonal responses to hypotensive haemorrhage with and without concomitant administration of ADM in both non-pregnant and pregnant conscious sheep, under controlled experimental conditions. 


\section{Materials and Methods}

The study was approved by the Animal Ethics Committee of the Christchurch School of Medicine. A total of 14 Coopworth ewes (Lincoln University Farm, Christchurch, New Zealand) were housed in an air-conditioned lightcontrolled room and received a standard laboratory diet (60 mmol sodium and $150 \mathrm{mmol}$ potassium per day). Six sheep were non-pregnant and eight were pregnant (late gestation). With the animal under general anaesthesia (induced by $17 \mathrm{mg} / \mathrm{kg}$ thiopentone sodium and maintained by a mixture of halothane, nitrous oxide and oxygen), a Konigsberg (P4.0) high-fidelity pressure-tip transducer was implanted in a carotid artery (Konigsberg Instruments, Pasadena, CA, USA) for direct measurement of arterial pressure and heart rate. Polyethylene catheters were placed in both jugular veins for subsequent infusions, haemorrhage, right atrial pressure (RAP) measurement and blood sampling. The animals were allowed to recover for at least 7 days before experiments commenced.

Each animal was studied twice, at least 2 days apart. On one occasion they received, in balanced random order, ADM in haemaccel (Behring, Marburg, Germany), and on the other (control) the same volume of haemaccel (vehicle) alone. Human ADM(1-52) was administered via a jugular catheter in a dose of $5.5 \mathrm{pmol} / \mathrm{kg}$ per min for a total of $90 \mathrm{~min}$. Thirty minutes after commencement of infusions, haemorrhage was performed by removal of $15 \mathrm{ml} / \mathrm{kg}$ blood over a $15 \mathrm{~min}$ period, from a jugular catheter. Blood was maintained sterile and heparinised until completion of the experiment, at which time it was returned to the sheep.

Arterial pressure and RAP measurements commenced 30 min before ADM or vehicle infusions by an online data acquisition system (Dataflow, Crystal Biotech, Hopkinton, MA, USA). Heart rate and pressure were digitally integrated over $30 \mathrm{~s}$ recording periods and data from four consecutive periods were averaged and recorded at preset intervals throughout the study.

Venous blood was drawn at preset intervals throughout the study day, starting 30 min before infusions. Blood was taken into chilled EDTA tubes, centrifuged, and plasma stored at $-80{ }^{\circ} \mathrm{C}$. The following peptides were measured by radioimmunoassay: ACTH (Donald 1977), ADM (Lewis et al. 1996), aldosterone (Lun et al. 1983), angiotensin II (Nicholls \& Espiner 1976), AVP (Sadler et al. 1983), corticotrophin releasing factor (CRF) (Ellis et al. 1990), cortisol (Lewis et al. 1992) and PRA (Dunn \& Espiner 1976).

\section{Statistics}

Results are expressed as means \pm S.E.M. Two-way analysis of variance (ANOVA) with time as a repeated measure was used to determine time and treatment differences between ADM and control arms of the study or group differences (non-pregnant compared with pregnant) between control arms of the studies. Statistical significance was assumed at $P<0 \cdot 05$. Where significant differences were identified by ANOVA, a priori Fisher's protected least square difference (LSD) tests were used to identify individual time-points that were significantly different from time-matched controls.

\section{Results}

Effects of pregnancy on baseline variables and response to haemorrhage

Plasma immunoreactive ADM concentrations remained close to the limit of detection for the assay during vehicle control infusions and there were no significant differences observed between groups in either baseline values or their response to haemorrhage as observed on the control day data (Fig. 1).

Baseline differences in haemodynamic parameters (Fig. 2) were apparent between the groups for heart rate (non-pregnant $82.3 \pm 7.6$ beats $/ \mathrm{min}$, pregnant $102 \pm$ 6.8 beats $/ \mathrm{min} ; P=0.02)$. Baseline mean arterial pressure (MAP) and packed cell volume (PCV) tended to be lower and RAP higher in pregnant sheep than in non-pregnant animals (all $0 \cdot 05<P<0 \cdot 1$ ). Comparison of control-day haemorrhage data demonstrated that the decrease in MAP (in both absolute and percentage terms) was augmented in the pregnant sheep (peak decrease $22 \mathrm{mmHg}(27 \%)$ ) compared with that in the non-pregnant group (peak decrease $17 \mathrm{mmHg}(19 \%) ; P<0 \cdot 001)$. In contrast, the reflex increment in heart rate was attenuated $(16 \%$ compared with $38 \%)$ in the pregnant sheep $(P=0.006)$. There was no significant difference in the RAP response between groups. PCV responses to haemorrhage differed between groups $(P<0 \cdot 001)$, with an initial increase followed by a later decrease in the non-pregnant group, but a steady reduction throughout the experiment in the pregnant sheep.

Baseline differences in the RAS and aldosterone were apparent between the groups, with PRA (nonpregnant $0.295 \pm 0.03 \mathrm{nmol} / 1$ per $\mathrm{h}$, pregnant $0.605 \pm$ $0.03 \mathrm{nmol} / 1$ per $\mathrm{h} ; P=0.02$ ), angiotensin II (non-pregnant $25 \cdot 3 \pm 1 \cdot 2 \mathrm{pmol} / \mathrm{l}$, pregnant $31 \cdot 5 \pm 2 \cdot 5 \mathrm{pmol} / 1 ; P=0 \cdot 05)$ and aldosterone (non-pregnant $240 \cdot 2 \pm 50 \cdot 3 \mathrm{pmol} / 1$, pregnant $688.3 \pm 83.3 \mathrm{pmol} / \mathrm{l} ; \quad P=0.001)$ all being increased in pregnancy (Fig. 3). The PRA $(P=0.027)$, plasma angiotensin II $(P<0.001)$ and aldosterone $(P<0 \cdot 001)$ responses to haemorrhage were all augmented in pregnant sheep compared with those in non-pregnant sheep.

There were no baseline differences in the HPA axis apparent between the groups (Fig. 4). However, comparison of control-day haemorrhage data demonstrates that the plasma $\operatorname{AVP}(P=0 \cdot 009)$ and ACTH $(P=0 \cdot 007)$ responses were both significantly greater in the pregnant sheep. 


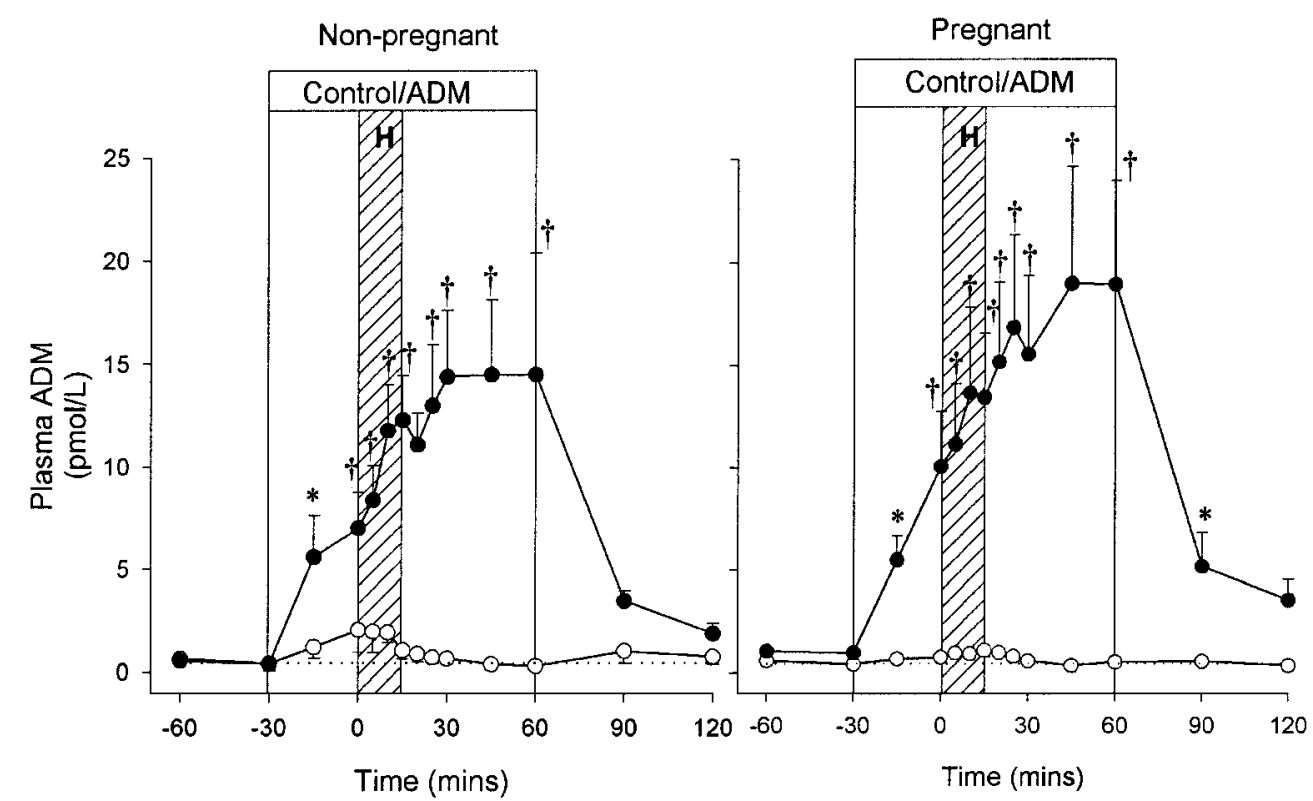

Figure 1 Plasma adrenomedullin (ADM) during infusion of ADM $(\bullet)$ at a dose of $5.5 \mathrm{pmol} / \mathrm{kg}$ per min or vehicle control $(O)$ in six non-pregnant sheep (left panel) and eight pregnant sheep (right panel), across haemorrhage $(\mathrm{H})$. Values shown are means \pm S.E.M. Dotted line represents level of detection in the assay. Plasma ADM concentrations were significantly increased in response to ADM infusions in both groups (both $P<0 \cdot 001$ ). Individual time points significantly different from time-matched control (Fisher's protected LSD from two-way ANOVA): ${ }^{*} P<0 \cdot 05,+P<0 \cdot 01$.

There were no significant differences in CRF or cortisol responses between the groups.

\section{Effects of $A D M$}

Plasma immunoreactive ADM concentrations increased similarly during peptide infusions in both nonpregnant and pregnant sheep (both $P<0 \cdot 001$ ), to plateau at approximately $15-20 \mathrm{pmol} / 1$ (Fig. 1).

There was no significant effect of ADM on the haemorrhage-induced decreases in MAP or RAP in either group (Fig. 2). Similarly, ADM altered neither heart rate nor PCV responses to haemorrhage.

The effect of ADM on the RAS response to haemorrhage was altered by pregnancy (Fig. 3). In non-pregnant sheep, haemorrhage-induced activation of PRA $(P=0 \cdot 005)$ and plasma angiotensin II $(P=0.037)$ were significantly augmented by ADM compared with control infusion. PRA was significantly increased in response to ADM before haemorrhage (time-point 0 ), with activity further enhanced in response to haemorrhage whereas augmentation of the plasma angiotensin II response was statistically significant only after haemorrhage. In contrast, the haemorrhage-induced activation of plasma aldosterone was not significantly altered by ADM. In pregnant sheep, there was no augmentation of PRA and plasma angiotensin II responses to haemorrhage. Furthermore, the plasma aldosterone response to haemorrhage was significantly attenuated by ADM $(P=0.039)$.

The effects of ADM on the HPA axis response to haemorrhage were also different between the groups (Fig. 4). In the non-pregnant sheep, ADM significantly augmented the AVP response to haemorrhage $(P=0 \cdot 003)$. The plasma ACTH response to haemorrhage tended to be greater during ADM infusion compared with control values, although this did not attain statistical significance. Haemorrhage induced similar increases in plasma CRF and cortisol, with no statistically significant differences between ADM and control treatments. However, in the pregnant sheep, ADM had no statistically significant effect on the AVP, CRF or cortisol responses to haemorrhage, whereas the plasma ACTH response was reduced compared with control $(P=0 \cdot 012)$.

\section{Discussion}

A variety of hormonal actions have been described for ADM, but few in vivo studies have examined the effects of physiologically relevant doses of ADM on hormonal axes in an integrated manner. Furthermore, there have been no such reports of ADM actions on hormonal axes in pregnancy. In the present study, plasma ADM concentrations increased during peptide infusions, to plateau at 


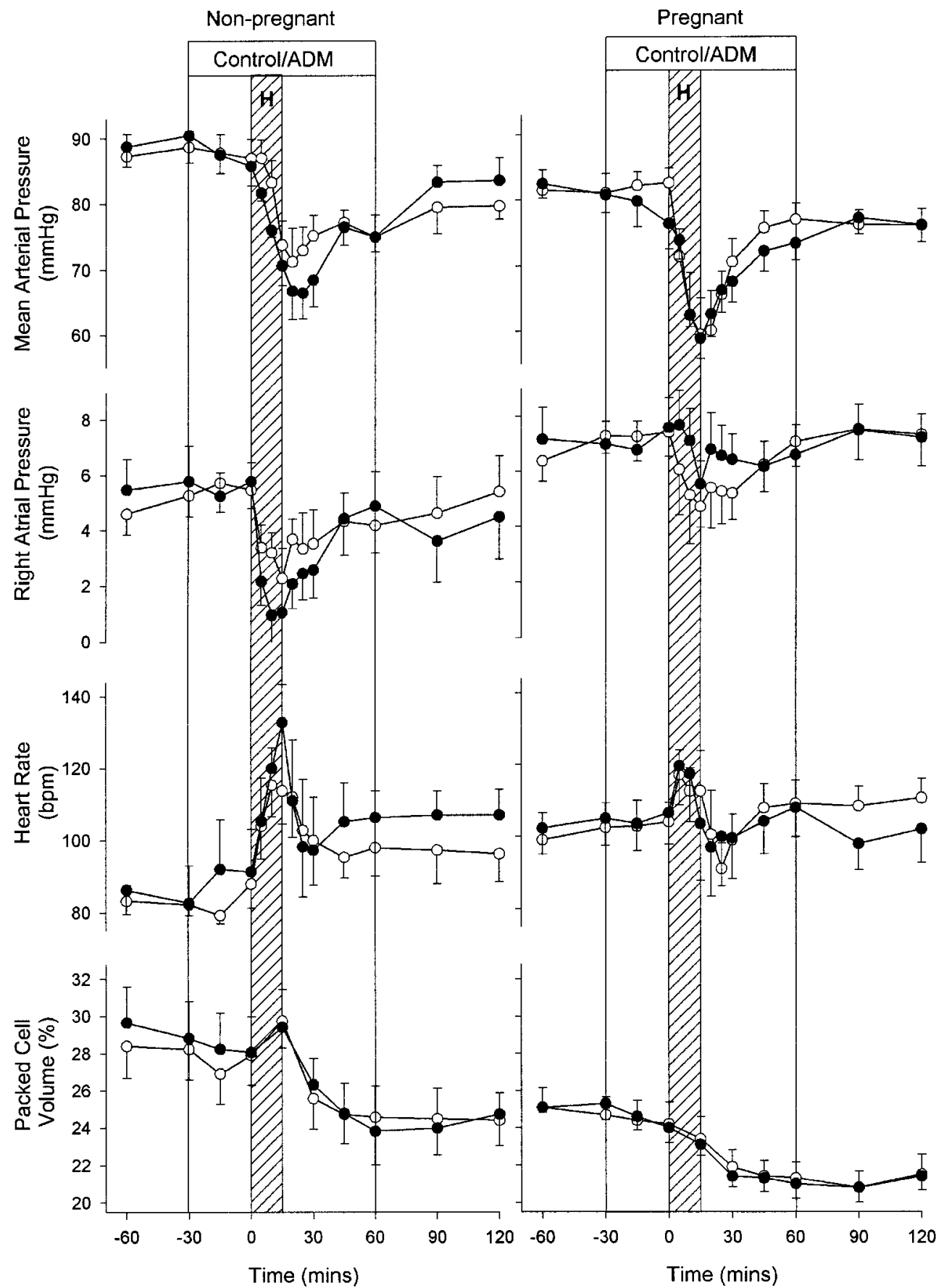

Figure 2 Haemodynamic response to haemorrhage $(\mathrm{H})$ during infusion of ADM $(\mathbf{})$ at a dose of $5.5 \mathrm{pmol} / \mathrm{kg} / \mathrm{min}$ and vehicle control $(\bigcirc)$ in six non-pregnant sheep (left panels) and eight pregnant sheep (right panels). Values shown are means \pm S.E.M.

approximately 15-20 pmol/1. Haemorrhage induced a greater decrease in MAP but a blunted increase in heart rate in pregnant sheep compared with non-pregnant ones. There was no significant effect of ADM on haemo- dynamic responses to haemorrhage in either group. Hypotensive haemorrhage was associated with significantly greater activation of the RAS in pregnant than in nonpregnant sheep. However, the effect of ADM on the RAS 


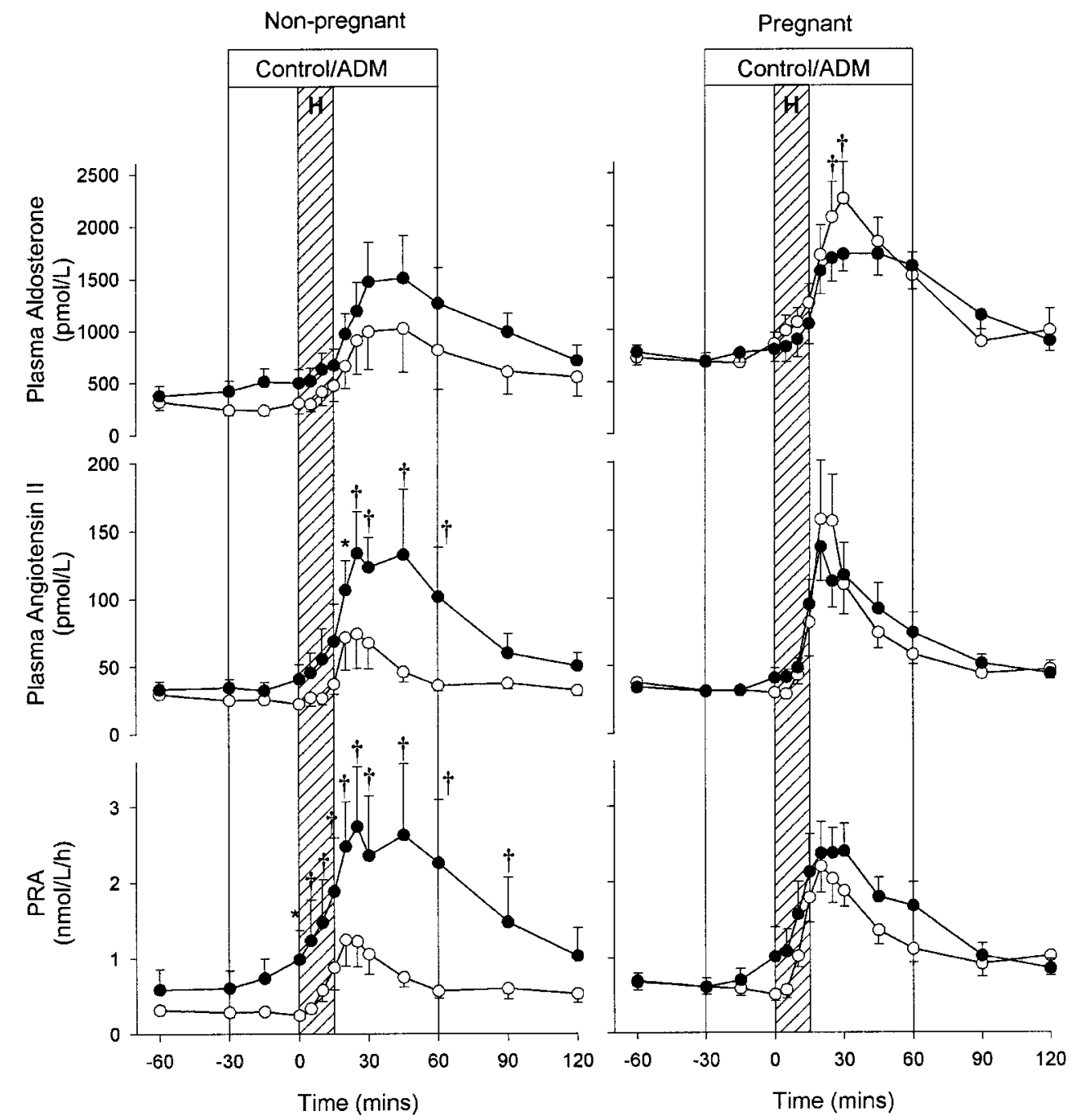

Figure 3 PRA, plasma angiotensin II and aldosterone responses to haemorrhage $(H)$ during infusion of ADM $(\mathbf{O})$ at a dose of $5.5 \mathrm{pmol} / \mathrm{kg}$ per min and vehicle control $(\bigcirc)$ in six non-pregnant sheep (left panels) and eight pregnant sheep (right panels). Values shown are means \pm S.E.M. Plasma angiotensin II $(P=0.037)$ and PRA $(P=0.005)$ concentrations were augmented during ADM compared with control infusion in the non-pregnant sheep. The plasma aldosterone $(P=0 \cdot 039)$ response was attenuated during ADM compared with control infusion in the pregnant sheep. Individual time points significantly different from time-matched control (Fisher's protected LSD from two-way ANOVA): ${ }^{*} P<0 \cdot 05,+P<0 \cdot 01$.

response was different between the two groups. In nonpregnant sheep, ADM augmented the RAS response to haemorrhage, as demonstrated by enhanced activation of PRA and plasma angiotensin II concentrations, but did not augment the plasma aldosterone response. In contrast, there was no augmentation of the RAS response to haemorrhage in pregnant sheep. Furthermore, the plasma aldosterone response was attenuated on the ADM day. As with the RAS response, the HPA axis (both plasma AVP and $\mathrm{ACTH}$ ) response to haemorrhage was greater in the pregnant sheep than in the non-pregnant sheep. Once again, the effect of ADM on the HPA response was different between groups. In non-pregnant sheep, the plasma AVP response was also augmented, whereas in pregnant sheep the plasma ACTH response was significantly attenuated by ADM.

Normal pregnancy is characterised by reductions in vascular resistance, arterial pressure and responses to endogenous vasoconstrictors. Conversely, cardiac output, heart rate and blood volume are increased. These changes are generally believed to be physiological adaptations that assure an adequate supply of blood to the fetus. The exact mechanism responsible for these changes remains unknown, although several locally produced vasoactive 


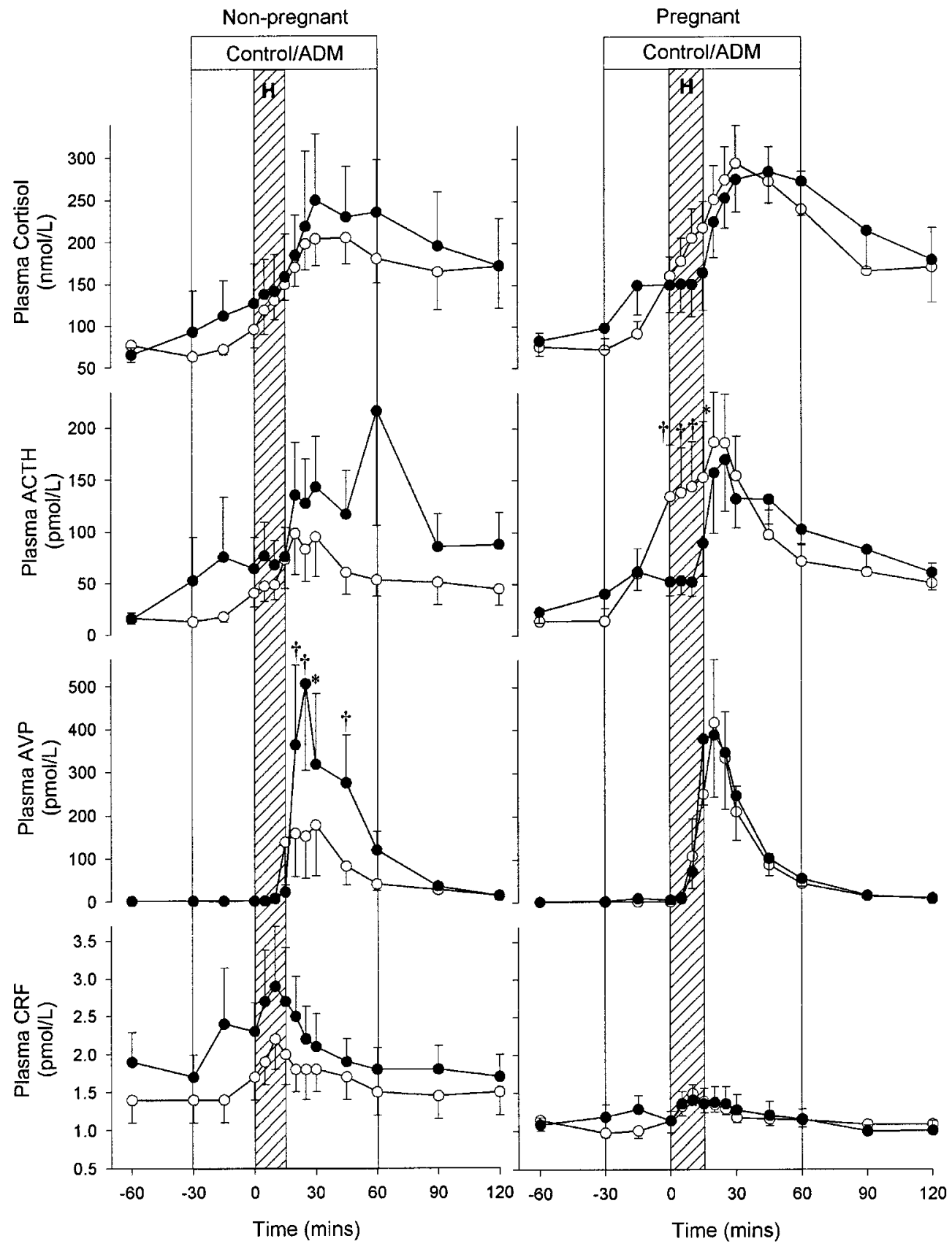

Figure 4 Plasma HPA axis response to haemorrhage $(\mathrm{H})$ during infusion of $\operatorname{ADM}(\mathbf{)})$ at a dose of $5.5 \mathrm{pmol} / \mathrm{kg} / \mathrm{min}$ and vehicle control $(O)$ in six non-pregnant sheep (left panels) and eight pregnant sheep (right panels). Values shown are means \pm S.E.M. AVP concentrations were augmented $(P=0.003)$ during ADM compared with control data in the non-pregnant sheep. Plasma ACTH concentrations $(P=0 \cdot 012)$ were attenuated during ADM compared with control data in the pregnant sheep. Individual time points significantly different from time-matched control (Fisher's protected LSD from two way ANOVA): ${ }^{*} P<0 \cdot 05$, $+P<0 \cdot 01$.

substances are implicated in the regulation of the fetoplacental circulation (Di Iorio et al. 1997, Jerat \& Kaufman 1998). It is also well established that pregnant animals are less able to maintain arterial pressure during haemorrhage than are non-pregnant animals (Brooks et al. 1995). This was seen in the present study, in which decreases in 
arterial pressure during standardised hypotensive haemorrhage were greater in pregnant sheep than in nonpregnant animals. Thus the ability to compensate for a reduction in blood volume is attenuated during pregnancy. There is also evidence of blunted baroreflex control of sympathetic activity in pregnancy (Brooks et al. 1995, Keller-Wood 1995) and this presumably explains the reduced heart rate response to haemorrhage in the pregnant sheep in the present study. Given the chronic alterations in blood volume and blood pressure - both variables that determine the secretion of a number of hormones such as the HPA and RAS - it is logical to hypothesise that the set-point for regulation of these hormones is reset in pregnancy. Indeed, it has been reported that AVP and ACTH responses to hypotension are reduced in pregnant ewes (Keller-Wood 1995). This contrasts with the findings of the present study, which presumably relates to the considerably greater decrease in MAP (in both absolute and percentage terms) observed in the pregnant sheep compared with that in the non-pregnant group.

That ADM infusions can induce an increase in PRA is well documented (Parkes \& May 1995, Charles et al. 1997); this is possibly the result of a decrease in arterial pressure stimulating the afferent renal arteriolar baroreceptor, but also possibly produced via activation of sympathetic activity to the kidney and a direct stimulatory effect on juxtaglomerular granular cells (Jensen et al. 1997). This was again apparent in the present study, in which 30 min of ADM infusion (time-point 0 ) had significantly increased the PRA, even before haemorrhage. Stimulation of PRA by ADM was accentuated after haemorrhage, with concentrations remaining some threefold greater than time-matched controls for the duration of the experiment. Such dramatic effects of ADM on PRA were paralleled by the augmentation of plasma angiotensin II. This highly significant augmentation of the RAS response by ADM occurred in the absence of significant differences in arterial pressure between ADM-treated and control animals, suggesting again that factors beyond renal perfusion pressure (especially increased sympathetic activity and direct actions on the juxtaglomerula apparatus) were operative.

Despite such dramatic increases in PRA and plasma angiotensin II, the haemorrhage-induced increase in plasma aldosterone concentrations was not significantly enhanced by ADM. Furthermore, in pregnant sheep, in which PRA and plasma angiotensin II responses to haemorrhage were not augmented by ADM, the aldosterone response to haemorrhage was actually reduced. Thus ADM may have acted to suppress angiotensin II-induced aldosterone secretion from the adrenal gland. Consistent with this premise are observations from in vitro studies that ADM inhibits angiotensin II and potassium- (but not ACTH-) induced secretion of aldosterone (Yamaguchi et al. 1994, Nussdorfer et al. 1997, Andreis et al. 1998). Recent in vivo studies in sheep (Salemi et al. 2000) and humans (Petrie et al. 2000), along with the results of the present study, support an action of ADM to suppress angiotensin II-induced aldosterone secretion. A further factor in the pregnant sheep was the inhibitory action of $\mathrm{ADM}$ on $\mathrm{ACTH}$. As the latter is a potent secretagogue of aldosterone, inhibition of ACTH by ADM might have contributed to the significant suppressive action of ADM on aldosterone that we observed.

ADM has inhibitory actions on the HPA axis under some experimental circumstances. Samson et al. (1995) reported that ADM suppressed ACTH secretion from pituitary cells in vitro, and ADM administered intravenously to conscious sheep reduced plasma cortisol and ACTH concentrations (Parkes \& May 1995). In the non-pregnant sheep of the present study, we saw no effect of ADM on plasma ACTH or cortisol concentrations or their response to haemorrhage. However, the plasma AVP response to haemorrhage was greatly augmented by ADM. Given that AVP is a known secretagogue for ACTH, we may have observed a balance of inhibition of ACTH by exogenous ADM and stimulation by increased endogenous AVP. Interestingly for pregnant sheep, in the absence of a measurable augmentation of the plasma AVP response to haemorrhage, plasma ACTH concentrations were significantly reduced by exogenous ADM, both before and during haemorrhage.

The mechanism behind augmentation of plasma AVP concentrations in the non-pregnant sheep in response to ADM infusion is unclear. It has been previously reported that intracerebroventricular administration of ADM inhibits both hyperosmolar and hypovolaemia-induced AVP in conscious rats (Yokoi et al. 1996). It is possible that the stimulus for augmentation of the AVP response to haemorrhage was the trend for a more precipitous decrease in arterial pressure with ADM. In fact, it may have been expected that the hypotensive response to haemorrhage would have been exacerbated by ADM and, thus, augmented AVP secretion served to counteract a further decrease in arterial pressure and ameliorated the decrease in blood pressure.

A number of studies have suggested that ADM may have a significant role in the cardiovascular adaptation to pregnancy (Di Iorio et al. 1997, Jerat \& Kaufman 1998, Shibata et al. 2000). It is unclear why ADM exhibited a different profile of actions to modulate the response to hypotensive haemorrhage in the two groups (nonpregnant and pregnant) in the present study. Interestingly, the hyperdynamic state of pregnancy characterised by increased plasma volume and decreased systemic vascular resistance is generally believed to contribute to a blunting of the pressor response to vasoactive substances such as angiotensin II (Paller 1984). We postulate that this same volume-replete hyperdynamic state contributed to a relative blunting of the actions of ADM to augment the AVP, PRA and angiotensin II response to hypotensive haemorrhage. Under such conditions, the actions of ADM in 
suppressing the aldosterone response to haemorrhage were unmasked. It has been reported previously that the hypotensive effect of ADM is potentiated in pregnant rats (Makino et al. 1999). Our study was not designed to examine this but, interestingly, arterial pressure appeared to decrease more precipitously in response to ADM before haemorrhage (time-point 0) in the pregnant sheep. However, this trend did not reach statistical significance.

One issue possibly complicating interpretation of our data is the redistribution of blood flow with pregnancy and the effects of exogenous infusions thereon, such as has been reported with prolonged infusion of oestrogen. Magness et al. (1998) reported increased blood flow to some non-reproductive beds (skin, heart, skeletal muscle, brain) and reproductive beds during 10 days of $17 \beta$-oestradiol infusions in ovariectomised sheep. Under these circumstances, haemorrhage with or without concurrent ADM infusion may result in contrasting alterations in blood flow to different organs, which probably contributed to both production and clearance of the hormones that we measured. Changes in blood flow, not measured in the present study, will ultimately be needed to interpret the hormone and haemodynamic responses that we recorded. For example, it is presumed that the ADM-related inhibition of the plasma aldosterone response to haemorrhage is accounted for by a direct suppressant effect of ADM on the adrenal glomerulosa; although this might be so, changes in regional blood flow to organs involved in the clearance of aldosterone, particularly the liver, could also contribute.

Because the amino acid sequence of ovine ADM has yet to be determined, we infused the human form in the present studies. There is a high degree of homology for ADM between species sequences thus far identified (Richards et al. 1996). However, it appears that bioactivity may be species-specific (Kohno et al. 1996), hence it will ultimately be important to determine the biological actions of the hormone in the species of origin. Using an assay developed for measuring ADM in human plasma (Lewis et al. 1996), we could not confirm detectable immunoreactive ADM in sheep plasma - endogenous concentrations were close to the limit of detection of the assay. Nevertheless, with the dose used in the present study, the achieved plasma concentrations of 15-20 pmol/l are at the upper limit of physiological for humans (normal range in our laboratory with this assay is $2 \cdot 7-10 \cdot 1 \mathrm{pmol} / \mathrm{l})$. Given that other investigators have reported that pregnancy increases plasma ADM concentrations three- to sixfold over those in non-pregnant controls (Di Iorio et al. 1997, 1999, Jerat \& Kaufman 1998, Minegishi et al. 1999), concentrations obtained in the present study are likely to be similar to those observed in normal pregnancy. One point of uncertainty in this discussion is the potential confounding effects of ADM binding proteins. Pio et al. (2001) describe the existence of a large-capacity ADM binding protein, complement factor $\mathrm{H}$ in human plasma. This factor can interfere with measurements of plasma ADM. Until the status of ADM-binding proteins in sheep is clarified, interpretation of the physiological and pathophysiological relevance of achieved ADM concentrations in our experiments remains uncertain.

In conclusion, ADM significantly augments the RAS and HPA axis response to hypotensive haemorrhage in non-pregnant, but not in pregnant, sheep. In contrast, it inhibits the ACTH and aldosterone responses to haemorrhage in pregnant sheep. This study provides further evidence for a potentially important role for ADM in modulating hormonal responses to stress, but its modulatory characteristics may differ between the pregnant and non-pregnant states.

\section{Acknowledgements}

We are grateful to staff of the Christchurch School of Medicine Animal Laboratory for assistance with animal studies and Christchurch Cardioendocrine Laboratory staff for hormone assays. Support was provided through grants from the National Heart Foundation of New Zealand, Lotteries Health Research and Health Research Council of New Zealand.

\section{References}

Andreis PG, Tortorella C, Mazzochi G \& Nussdorfer GG 1998 Proadrenomedullin N-terminal 20 peptide inhibits aldosterone secretion from human adrenocortical and Conn's adenoma cells: comparison with adrenomedullin effect. Journal of Clinical Endocrinology and Metabolism 83 253-257.

Brooks VL, Quesnell RR, Cumbee SR \& Bishop VS 1995 Pregnancy attenuates activity of the baroreceptor reflex. Clinical and Experimental Pharmacology and Physiology 22 152-156.

Cameron VA \& Fleming AM 1998 Novel sites of adrenomedullin gene expression in mouse and rat tissues. Endocrinology 139 2253-2264.

Caron KM \& Smithies O 2001 Extreme hydrops fetalis and cardiovascular abnormalities in mice lacking a functional adrenomedullin gene. PNAS 98 615-619.

Charles CJ, Rademaker MT, Richards AM, Cooper GJS, Coy DH, Jing NY \& Nicholls MG 1997 Hemodynamic, hormonal, and renal effects of adrenomedullin in conscious sheep. American Journal of Physiology 272 R2040-R2047.

Di Iorio R, Marinoni E, Scavo D, Letizia C \& Cosmi EV 1997 Adrenomedullin in pregnancy. Lancet 349328.

Di Iorio R, Marinoni E, Letizia C, Villaccio B, Alberini A \& Cosmi EV 1999 Adrenomedullin production is increased in normal human pregnancy. European Journal of Endocrinology 140 201-206.

Donald RA 1977 Radioimmunoassay of corticotropin (ACTH). In Handbook of Radioimmunoassay, pp 319-390. Ed G Abrahams. New York and Basel: Marcel Dekker.

Dunn PJ \& Espiner EA 1976 Outpatient screening tests for primary aldosteronism. Australian and New Zealand Journal of Medicine 6 131-135.

Ellis MJ, Schmidli RS, Donald RA, Livessey JH \& Espiner EA 1990 Plasma corticotrophin releasing factor and vasopressin 
responses to hypoglycaemia in normal man. Clinical Endocrinology 32 93-100.

Jensen BL, Kramer BK \& Kurtz A 1997 Adrenomedullin stimulates renin release and renin mRNA in mouse juxtaglomerular granular cells. Hypertension 29 1148-1155.

Jerat S \& Kaufman S 1998 Effect of pregnancy and steroid hormones on plasma adrenomedullin levels in the rat. Canadian Journal of Physiology and Pharmacology 76 463-466.

Keller-Wood M 1995 Reflex regulation of hormonal responses during pregnancy. Clinical and Experimental Pharmacology and Physiology 22 143-151.

Kohno M, Yasunari K, Yokokawa K, Horio T, Ikeda M, Kano H, Minami M, Hasnehira T \& Yoshikawa J 1996 Interaction of adrenomedullin and platelet-derived growth factor on rat mesangial cell production of endothelin. Biochemical and Biophysical Research Communications 194 720-725.

Lainchbury JG, Cooper GJS, Coy DH, Jing NY, Lewis LK, Yandle TG, Richards AM \& Nicholls MG 1997 Adrenomedullin: a hypotensive hormone in man. Clinical Science 92 467-472.

Lewis JG, Manley L, Whitlow JC \& Elder PA 1992 Production of a monoclonal antibody to cortisol: application to a direct immunosorbent assay of plasma. Steroids $\mathbf{5 7} 82-85$.

Lewis LK, Smith MW, Charles CJ, Lainchbury JG, Yandle TG, Richards AM \& Nicholls MG 1996 Plasma assay and in vivo kinetics of human adrenomedullin (1-52) in humans and sheep. Proceedings of the 10th International Congress of Endocrinology, San Francisco, CA, USA. Abstract pp 1-768.

Lun S, Espiner EA, Nicholls MG \& Yandle TG 1983 A direct radioimmunoassay for aldosterone in plasma. Clinical Chemistry 29 268-271.

Magness RR, Phernetton TM \& Zheng J 1998 Systemic and uterine blood flow distribution during prolonged infusion of $17 \beta$-estradiol. American Journal of Physiology 275 H731-H743.

Makino I, Shibata K, Makino Y, Kangawa K \& Kawarabayashi T 1999 Potentiation of the hypotensive effect of adrenomedullin in pregnant rats. European Journal of Pharmacology 385 129-136.

Minegishi T, Nakamura M, Abe K, Tano M, Andoh A, Yoshida M, Takagi T, Nishikimi T, Kojima M \& Kangawa K 1999 Adrenomedullin and atrial natriuretic peptide concentrations in normal pregnancy and pre-eclampsia. Molecular Human Reproduction $5767-770$.

Nicholls MG \& Espiner EA 1976 A sensitive, rapid radioimmunoassay for angiotensin II. New Zealand Medical Journal 83 399-403.

Nussdorfer GG, Rossi GP \& Mazzocchi G 1997 Role of adrenomedullin and related peptides in the regulation of the hypothalamopituitary-adrenal axis. Peptides 18 1079-1089.

Paller MS 1984 Mechanism of decreased pressor responsiveness to ANG II, NE, and vasopressin in pregnant rats. American Journal of Physiology $261 \mathrm{H} 100-\mathrm{H} 108$.
Parkes DG \& May CN 1995 ACTH-suppressive and vasodilator actions of adrenomedullin in conscious sheep. Journal of Neuroendocrinology 7 923-929.

Petrie MC, Hiller C, Morton JJ \& McMurray JJ 2000 Adrenomedullin selectively inhibits angiotensin II-induced aldosterone secretion in humans. Journal of Hypertension 18 61-64.

Pio R, Martinez A, Unsworth EJ, Kowolak JA, Bengoechea JA, Zipfel PF, Elsasser TH \& Cuttitta F 2001 Complement factor $\mathrm{H}$ is a serum-binding protein for adrenomedullin and the resulting complex modulates the bioactivities of both partners. Journal of Biological Chemistry 276 2292-12300.

Rademaker MT, Charles CJ, Lewis LK, Yandle TG, Cooper GJS, Coy DH, Richards AM \& Nicholls MG 1997 Beneficial hemodynamic and renal effects of adrenomedullin in an ovine model of heart failure. Circulation 96 1983-1990.

Richards AM, Nicholls MG, Lewis L \& Lainchbury JG 1996 Adrenomedullin. Clinical Science 91 3-16.

Sadler WA, Lynskey C, Gilchrist N, Espiner EA \& Nicholls MG 1983 A sensitive radioimmunoassay for measuring plasma antidiuretic hormone in man. New Zealand Medical Journal 96 959-963.

Salemi R, McDougall JG, Hardy KJ \& Wintour EM 2000 Effect of adrenomedullin infusion on basal and stimulated aldosterone secretion in conscious sheep with cervical adrenal autotransplants. Journal of Endocrinology 166 389-399.

Samson W, Murphy T \& Schell D 1995 A novel vasoactive peptide, adrenomedullin, inhibits pituitary adrenocorticotropin release. Endocrinology 136 2349-2352.

Shibata K, Makino I, Makino Y, Kangawa K \& Kawarabayashi T 2000 A possible role of adrenomedullin in the altered cardiovascular reactivity of pregnancy in the rat [abstract]. Proceedings of the 2nd International Symposium on Adrenomedullin and PAMP, Miyazaki, Japan, p. 74.

Upton PD, Austin C, Taylor GM, Nandah KA, Clark AJL, Ghatei MA, Bloom SR \& Smith DM 1997 Expression of adrenomedullin $(A D M)$ and its binding sites in the rat uterus: increased number of binding sites and ADM messenger ribonucleic acid in 20-day pregnant rats compared with nonpregnant rats. Endocrinology 138 2508-2514.

Yamaguchi T, Baba Y, Doi Y \& Yano K 1994 Effect of adrenomedullin in aldosterone secretion by dispersed rat adrenal zona glomerulosa cells. Life Science 56 379-387.

Yokoi H, Arima H, Murase T, Kondo K, Iwasaki Y \& Oiso Y 1996 Intracerebroventricular injection of adrenomedullin inhibits vasopressin release in conscious rats. Neuroendocrinology Letters 216 65-67.

Received in final form 4 July 2001 Accepted 9 July 2001 